\title{
Adrian VELICU, Civic Catechisms and Reason in the French Revolution
}

Jean-Charles Buttier

\section{(2) OpenEdition \\ Journals}

Édition électronique

URL : https://journals.openedition.org/ahrf/12387

DOI : 10.4000/ahrf.12387

ISSN : 1952-403X

Éditeur :

Armand Colin, Société des études robespierristes

Édition imprimée

Date de publication : 1 mars 2012

Pagination : 228-230

ISSN : 0003-4436

Référence électronique

Jean-Charles Buttier, «Adrian veucu, Civic Catechisms and Reason in the French Revolution », Annales historiques de la Révolution française [En ligne], 367 | janvier-mars 2012, mis en ligne le 12 septembre 2012, consulté le 01 juillet 2021. URL : http://journals.openedition.org/ahrf/12387 ; DOI : https:// doi.org/10.4000/ahrf.12387

Ce document a été généré automatiquement le 1 juillet 2021.

Tous droits réservés 


\title{
Adrian VELICU, Civic Catechisms and Reason in the French Revolution
}

\author{
Jean-Charles Buttier
}

\section{RÉFÉRENCE}

Adrian VELICU, Civic Catechisms and Reason in the French Revolution, Farnham, Ashgate, 2010, 180 p., ISBN 978-0-7546-6998-2, $80 €$.

1 Adrian Velicu a choisi d'étudier en parallèle les catéchismes politiques édités pendant la Révolution française et l'usage du concept de Raison à cette période. L'auteur, maître de conférences au département d'Histoire des Idées de l'Université de Karlstad, n'est pas un spécialiste de la Révolution française. Il avait cependant déjà étudié ce genre de la littérature politique dans un article de 2001: Civic catechisms and Reason during the French Revolution. La publication de cet ouvrage est l'occasion de reprendre et de développer l'idée que les catéchismes révolutionnaires révèlent par leur dimension oxymorique une contradiction profonde de la Révolution qui peut se résumer par l'expression «émanciper par la catéchèse » (p. 14). Il met en effet en parallèle la Raison émancipatrice et les projets éducatifs fondés selon lui sur l'endoctrinement et sur l'inculcation d'une foi nouvelle. L'hypothèse plus générale est d'illustrer le contraste entre l'utilisation d'une rhétorique emprunte de religiosité, en particulier en l'an II, et l'idéal de rationalité perçu comme élément constitutif de la Révolution. Adrian Velicu a mobilisé trois types de sources pour son étude : les discours d'assemblées et projets de lois relatifs à l'éducation d'un côté et les catéchismes civiques de l'autre. La bibliographie de l'ouvrage comporte 41 catéchismes civiques.

Dans un premier chapitre intitulé Catechisms : A Retrospective, l'auteur rappelle l'histoire du genre catéchisme depuis l'Antiquité jusqu'à la Révolution française en faisant d'utiles rappels sur la catéchèse protestante. Le deuxième chapitre (Reason: Usage and Meaning) est l'occasion d'étudier à rebours le concept de Raison en partant des années 1790 et en remontant jusqu'au XVII ${ }^{e}$ siècle. Adrian Velicu a choisi de mener ces deux 
études en parallèle afin de contextualiser les liens qui se nouent entre ces notions pendant la Révolution. Ensuite, l'auteur consacre un troisième chapitre (In Two Minds : Constitutional Monarchy) à l'analyse proprement dite des discours, projets et catéchismes écrits dans le contexte de la réunion des États généraux et de la monarchie constitutionnelle. C'est l'occasion d'expliquer l'émergence de ce genre littéraire par l'entrecroisement des sphères politique, religieuse et scolaire. Les chapitres 4 et 5 (Republican Questions. Republican Answers) concernent la période républicaine et plus particulièrement l'an II puisque l'historien décrit la forte production de catéchismes à cette période. Le chapitre 4 s'intéresse plus précisément à l'opposition entre idéal d'émancipation intellectuelle et régénération civique : «intellectual emancipation or civic renewal by ideological discipline? » (p. 90). Pour l'auteur le choix même de la forme catéchistique démontre l'importance accordée à une éducation politique qui exclut tout jugement critique. Dans le chapitre 5, Adrian Velicu voit dans l'explosion éditoriale de l'an II et dans le développement des catéchismes copiant le modèle chrétien la preuve que le choix même d'écrire un catéchisme est le reflet de la volonté de refuser tout esprit critique. D'ailleurs cette période se révèle dans l'expression oxymorique de "catéchisme révolutionnaire» qui résumerait la recherche contradictoire d'ordre dans le désordre révolutionnaire. Enfin, le chapitre 6 s'intéresse à la reprise de ces questions fondamentales après la chute de Robespierre (After Thermidor) et insiste sur la continuité de la politique éducative du Directoire comme le montre le prix reçu par le Catéchisme républicain, philosophique et moral de Poisson de La Chabeaussière à l'issue du concours pour la rédaction de livres élémentaires lancé en l'an II mais dont les résultats ne sont publiés qu'en l'an IV.

3 Il aurait été intéressant de connaître les critères de choix de la quarantaine de catéchismes politiques cités, à rapporter aux 151 catéchismes politiques différents publiés entre 1789 et 1799 , pour un total de 355 éditions. D'autre part, il aurait été utile d'avoir des données quantitatives et une typologie des catéchismes révolutionnaires comme a pu le proposer Jean Hébrard dans ses articles. L'absence de données matérielles peut s'expliquer par le fait qu'Adrian Velicu définisse d'abord ces textes comme une « constellation d'indices» (p. 153). Ces indices font sens par leur existence même et ne doivent pas être considérés comme des preuves, inutiles selon lui pour sa démonstration: "In a sense, the present discussion has inadvertently resorted to Robespierre's legal method to be applied in exceptional circumstances: «indices» (indications, clues) suffice, preuves (hard evidence) are not necessary " (p. 153). Outre les questions méthodologiques qu'une telle remarque soulève, l'absence d'analyse quantitative et matérielle de ces ouvrages ne permet pas d'étudier leur réception. L'auteur aurait pu utilement suivre les pistes lancées par Bernard Plongeron dans son analyse de la catéchèse chrétienne pour aborder la triade producteur / intermédiaire / récepteur. Toutefois, Adrian Velicu revendique une analyse purement textuelle en la replaçant dans la filiation des travaux de François Furet qu'il dit dépasser par cette analyse post-révisionniste (p. 11). Cette production doit toutefois être replacée dans un contexte de production, chronologique comme géographique, plus large sur le genre du catéchisme politique. Ainsi, la bibliographie ne fait pas référence aux travaux les plus récents sur le genre catéchisme politique présentés dans Les catéchismes républicains, numéro daté de septembre 2009 de la revue électronique $L a$ Révolution française. Enfin, l'ouvrage Civic Catechisms and Reason during the French Revolution contient des remarques téléologiques révélant une vision extrêmement négative de la Révolution française en général, et du catéchisme politique en 
particulier : «Embryonically, they also contained one of the less savoury components of modern political culture » (p. 151). 